\title{
Beyond the guidelines for the treatment of severely malnourished children with complications-assessment from Cambodia
}

\author{
Sophonneary Prak ${ }^{1}$, Miriam luell Dahl ${ }^{2}$, Joel Conkle ${ }^{2}$, Sam Oeurn Un ${ }^{2}$ and Arnaud Laillou ${ }^{2 *}$
}

\begin{abstract}
Background: From 2005 to 2010, the percentage of children dangerously thin (also called wasted) crossed the International Phase Classification Emergency Threshold, increasing from 8.4 to $10.9 \%$. As a result, Cambodia is now among the 20 worst countries globally for child wasting - with malnutrition implicated in more than 6400 child deaths annually. This study, therefore, sought to provide information on the actual treatment of severely acute malnourished children with complication in Cambodian hospitals.
\end{abstract}

Methods: Data was collected from March to June 2014, from hospital records of 1450 children admitted for treatment of severe acute malnutrition with complications as in-patient in 19 hospitals from 2011 to 2013. Recovery rates were determined using weight-for-height $z$-scores and the percentage of weight gain for children without oedema. Additional data on duration of stay in hospital, prevalence of follow-up visits, and rates of weight gain were also analyzed.

Results: According to weight for height z-score (WHZ), $58.6 \%$ of the children without oedema $(n=1100)$ were defined as cured of severe acute malnutrition at discharge. On average, during their stay in hospital, children were gaining approximately $7 \%$ of their bodyweight. In regards to follow-up visits, $86.7 \%$ of children without oedema were defined as cured after three follow-up visits, with an improved WHZ of $+2.28 z$-score since admission. When looking at the progress and achievements in regards to follow-up visits, the rate of children participating in three follow-up visits increased from 25.7 to $42.8 \%$ between 2011 and 2013.

Conclusions: A major finding from this research was that the children who completed three follow-up visits were not only cured ( $>-3$ standard deviation (SD)) but were no longer moderately malnourished, as most of them ended up with a $z$-score $>-2 S D$.

Keywords: SAM treatment, Cure rate, Cambodia, Guidelines, Malnutrition with complication

\section{Background}

Acute malnutrition continues to be a major public health concern worldwide, and in Southeast Asia alone, over two million children under the age of five are suffering from severe acute malnutrition (SAM) [1].

Cambodia is among the 20 worst countries globally when it comes to child malnutrition [2]. The Cambodian burden of undernutrition among children is enormous with $40 \%$ of children under the age of five stunted in 2010, $11 \%$ wasted, and $28 \%$ underweight [3]. Although

\footnotetext{
* Correspondence: alaillou@unicef.org

${ }^{2}$ UNICEF, Maternal, Newborn and Child Health and Nutrition section, No. 11 street 75, 12202 Phnom Penh, Cambodia

Full list of author information is available at the end of the article
}

stunting has decreased over the last decade, from $43 \%$ in 2005 and $50 \%$ in 2000, wasting has slightly increased since 2005. Malnutrition is implicated in more than 6400 child deaths annually, and child malnutrition has health and economic consequences that will affect Cambodia for generations to come [4]. Improving the nutritional status of children is therefore a development priority. Systematic poverty and poor child health continue to remain key issues in Cambodia [3], and an immediate reason for the persistence of child malnutrition is that over half of the children aged 6-23 months do not receive a minimum acceptable diet (United Nations children's fund (UNICEF) personal communication). 
Despite the relatively high prevalence of nutritional problems, there has been a lack of published data on the treatment of children with acute malnutrition in Southeast Asia [1]. With a prevalence of $2.5 \%$ of severely acute malnourished children in Cambodia [3], 60,000 to 80,000 cases could need specialized medical treatment, including therapeutic feeding, every year. Therefore, in Cambodia, UNICEF focused its intervention on treating cases of severe acute malnutrition (SAM) at the hospital level for those who have complications. Cambodia Hospital-based management of severe acute malnutrition started in the early 2000s with support from the Health Sector Support Project and UNICEF. Between 2008 and 2014, the hospitals implementing these services further doubled and in 2012, 35 out of 73 hospitals were able to provide SAM treatment of which 19 had more than 3 years of experiences. Guidelines for SAM management were revised in 2006 and later in 2012 with support from the Millennium Development Goal Funds (MDGF) program [5]. The treatment for malnutrition was normally carried out by one of the doctors or nurses at the hospital, as there was no staff position regarding nutrition at the referral hospital. Once discharged, the in-patient treatment of SAM cases with complications was followed up through out-patient visits to the hospital. This out-patient treatment was based on takehome rations with three follow-up visits.

Although programs have been in place to treat malnutrition since 2000, it is thought that program success is weakened by a high proportion of children not completely recovering before dropping out. These children are then likely to become severely malnourished again and are at a great risk of irreversible stunting because they would not return to a normal growth pattern [6]. All children, regardless of ethnicity, have the same potential for growth in the first 5 years of life. Until the age of five, height and weight are not greatly dependent on genetics [7]. It is estimated that proper identification and management of acute malnutrition could prevent over 400,000 child deaths in the world annually [8]. The actual paper is not evaluating the management of acute malnutrition and any preventing measures. The current study aimed to evaluate the treatment of severely acute malnourished children referred to hospital facilities as in-patient in Cambodia. The study will estimate the number of children cured at the end of the national protocol and will potentially also identify bottlenecks and way forward to improve national policies and guidelines to make it more efficient.

\section{Methods}

\section{Study design and inclusion criteria}

In addition to on-going monitoring data collected on a monthly basis by the National Nutrition Programme at the 35 hospitals, clinical records of children up to 6 years of age, admitted for treatment of acute malnutrition from 2011 to 2013, were collected from March to June 2014 in 19 hospitals from 13 provinces in Cambodia (Additional file 1: Figure S1). Children were admitted as in-patient treatment at the hospital if (i) there was bilateral pitting oedema, (ii) weight for height was less than -3 standard deviation, (iii) infants were $<6$ months with bilateral pitting oedema or visible wasting, or (iv) infants were $>6$ months but weigh less than $4 \mathrm{~kg}$.

The hospitals were selected according to the number of years of intervention. Hospital selected should have been implementing SAM treatment for more than 3 years. The study profile and the information collected are presented in Fig. 1. Recovery rates were determined using weightfor-height $z$-scores and the percentage of weight gain for children without oedema. Children with oedema were not included in our study, only the length of treatment at the hospital was observed. Additional data on duration of stay in hospital, prevalence of follow-up visits, and rates of weight gain were also analyzed. Data entering and staff interviews were conducted by UNICEF staff and a representative from the National Nutrition Programme (NNP). According to the Cambodian Ministry of Health, the study did not require a formal ethics approval as no name and no personal information have been collected.

\section{Data and statistical analysis}

The article was quality assessed using the STROBE combined checklist for case-control studies. Data was entered in Microsoft Excel 2013. For children from 6 months to 6 years, anthropometric $z$-scores were calculated using the National Center for Health Statistics/ WHO growth reference data of 2006 [9], using the WHO Anthro software for standard population. Anthropometric status was assessed by the following indicators: weight-forage $<-2 \quad z$-scores (WAZ) for underweight and weight-forheight $<-2 \mathrm{z}$-scores (WHZ) for wasting. Data management and analysis were performed with SPSS software version $20^{\text {mix }}$ (SPSS, V20; IBM SPSS, USA). Comparisons between groups (boys and girls) of data were performed using oneway analysis of variance (ANOVA).

\section{Results}

According to the on-going government monitoring, between 2011 and 2013, 4350 children were treated as inpatients for severe acute malnutrition. In the same period, the dropout and mortality rates decreased from 11.0 to $4.9 \%$ and 1.2 to $1.0 \%$, respectively (2011 as compared to 2013). In addition, when looking at the progress and achievements in regard to the out-patient treatment, the rate of children participating in three follow-up visits increased from 25.7 to $42.8 \%$ between 2011 and 2013 (Fig. 2). 

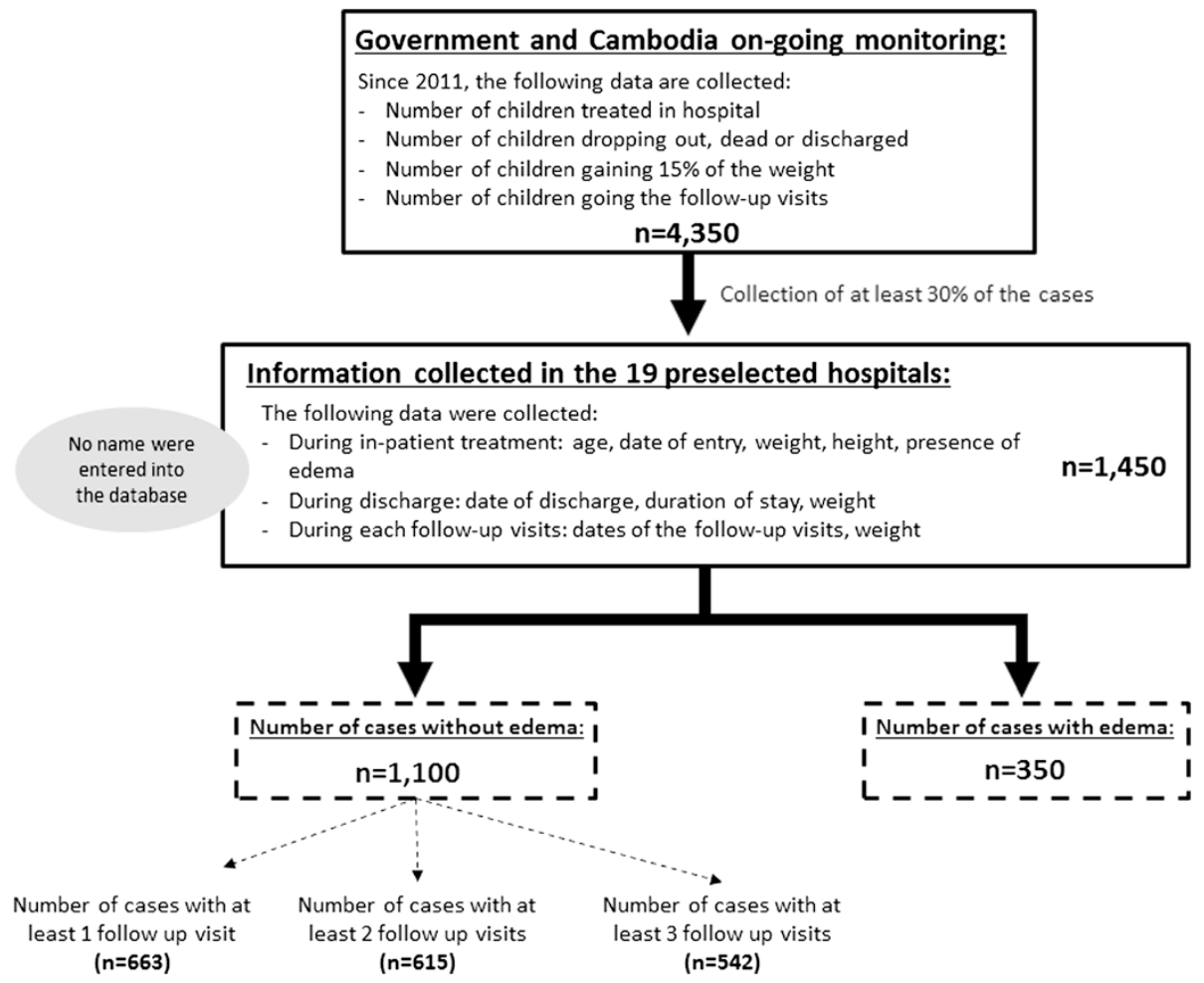

Fig. 1 Study profile

Among the cases collected $(n=1450), 50.9 \%$ were male and $49.1 \%$ female. The average age of the children treated was 17.1 months. The average duration of stay of children without oedema in hospital was $8.7 \pm 5.8$ days compared to $11.9 \pm 9.8$ days for children with oedema.

For children without oedema $(n=1100)$, overall bodyweight increased throughout the treatment period (from approximately 7.1 to $8.6 \mathrm{~kg}$ ) from admission to discharge and through follow-up 1, 2, and 3, respectively (Fig. 3). In average, the time between discharge and the first follow-up visit is 9.7 days, 15.2 days between the 1st follow-up visit and the second one and finally 19.9 days between the second and the third follow-up visit. When looking at the percentage of weight gain, the greatest

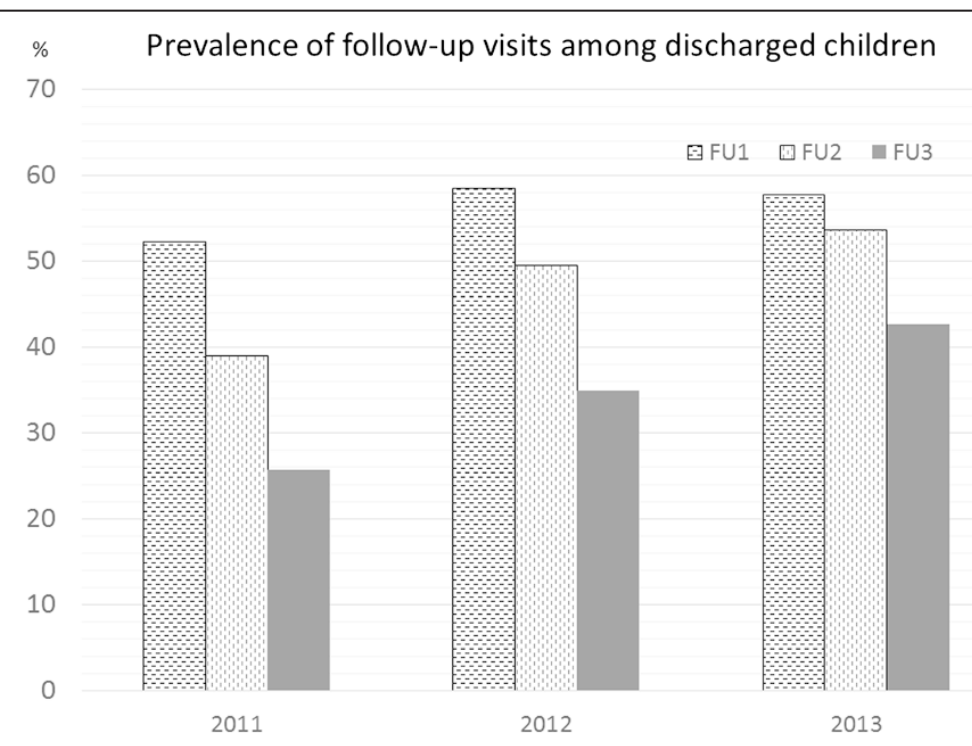

Fig. 2 Prevalence of follow-up visits among discharged children from 2011, 2012, and 2013 respectively 


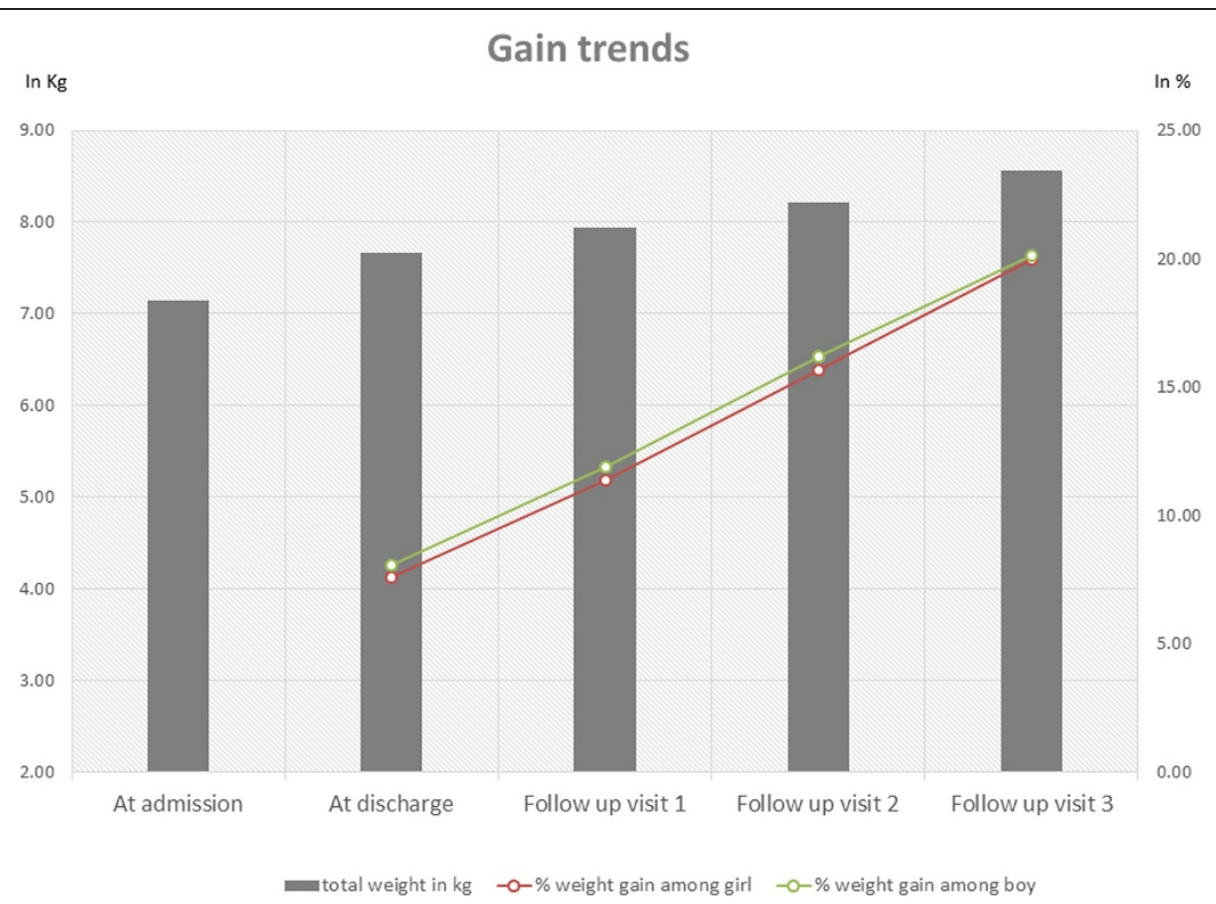

Fig. 3 Weight trends in kilograms (left axis) and percentage of weight gain (right axis) from admission to discharge and through follow-up 1, 2, and 3

increase was seen during in-patient treatment (IPD), with $7.6 \%$ for girls and $8.0 \%$ for boys $(p>0.05)$. In regard to both in-patient and out-patient treatment (three follow-up visits), the overall average weight gain was approximately $20 \%$ for both genders. In general, $58.6 \%$ of the children without oedema were defined as cured of severe acute malnutrition at discharge.

In regard to the follow-up visits, 75.6, 77.9, and $86.7 \%$ of children without oedema were defined as cured after one, two, and three follow-up visits, respectively, with an improved WHZ of approximately $+1.3 z$-score since admission after one follow-up visit, $+1.8 z$-score after two follow-up visits, and $+2.3 z$-score after three followup visits (Fig. 4). On average, the total duration of the treatment (IPD and three follow-up visits) lasted for a total of 53.5 days. All WAZ $z$-scores improved as well over the entire treatment period from $-3.67 z$-score to $-2.57 z$-score.

\section{Discussion}

This study showed that most of the children admitted to the hospital with SAM $<-3 \mathrm{SD}$, were actually discharged

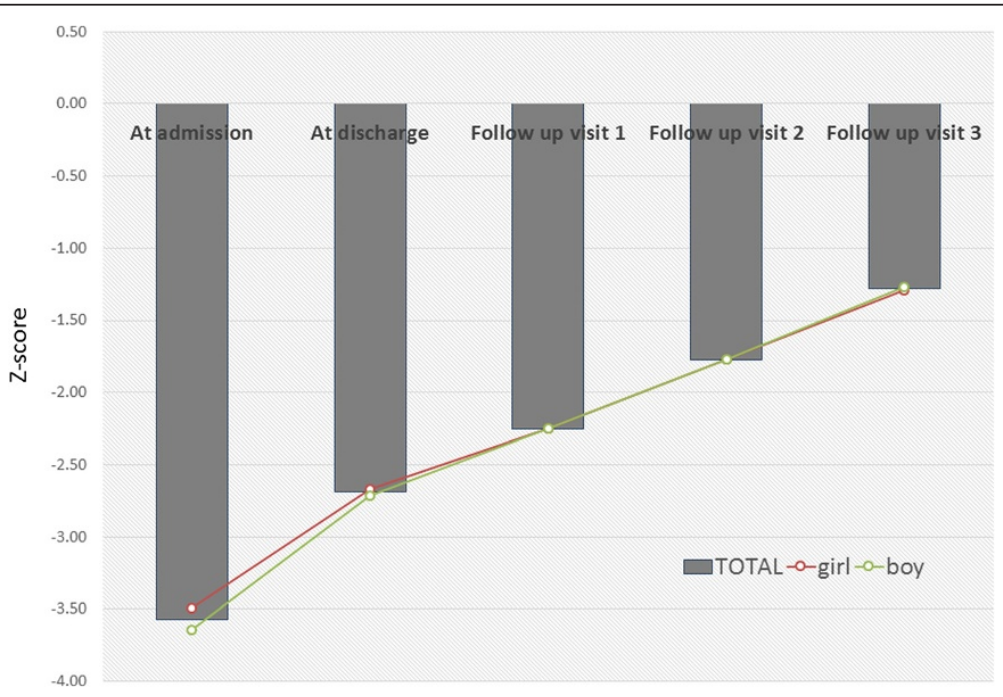

Fig. 4 Increase in WHZ (z-score in right axis) from admission to follow-up $3(n=542)$ 
as cured when using WHZ as an indicator (>-3SD). The latest report from the on-going monitoring from the government (personal communication) stated that $>65 \%$ of SAM children were not cured if the threshold of $15 \%$ weight gain was used; however, our results indicate that the proportion is substantially lower, with $41.4 \%$ not defined as cured at discharge. According to the current guidelines [5], the period of in-patient treatment is meant to last until the child has gained $15 \%$ of their own bodyweight; however, we found that the average duration of stay in the hospital was 8.7 days $( \pm 5.8)$, with an average weight increase of only $7.4 \%$. This finding indicates that there is a need to revise the current guidelines, which would make the management and treatment of SAM more efficient and cost-effective for the government as well as the caretakers. As highlighted by the 2013 WHO update on the treatment of severe acute malnutrition, $15 \%$ weight gain is not an appropriate discharge criterion [10]. This report emphasizes that the anthropometric indicator that is used to confirm severe acute malnutrition should also be used to assess whether a child has reached nutritional recovery [10].

One of the major findings from this research was that the children who completed three follow-up visits were not only cured based on the above -3SD but were also no longer moderately malnourished, as most of them ended up >-2SD.

Mothers staying in average of 8.7 days in the hospital are already an achievement in Cambodia, as most mothers and caretakers proved unwilling to stay at the hospital for much longer, due to reasons like responsibility for other children at home and the need to tend to farm and livestock. Additionally, research on dropping out by Noij \& Un (UNICEF personal communication) found that after the complementary disease was treated, mothers were unwilling to stay for malnutrition only, as her child could walk and play again and "did no longer look ill". However, mothers are increasingly bringing back their children to follow-up visits. There has been an increase in follow-up visits over the last years, and compliance for the third follow-up has increased by $40 \%$ from 2011 to 2013. To ensure that this compliance continues to increase, we need to ensure that mothers or caregivers are motivated. It appears from the study of Noij \& Un that compliance in Cambodia depend much on the motivation of the mother/caretaker which was in many cases not very high. With a limited motivation of mothers and caretakers, there does not need to be much of a reason to discontinue treatment and many small reasons were provided to justify default. The reasons to stop treatment included the taste of the therapeutic food but also the limited time to devote to the treatment as they needed to focus on other household and economic activities.
The substantial weight gain of most children using BP100 can be considered to provide a stimulus for caretakers to come for follow-up visits, as they see that the treatment does provide beneficial results for the child concerned. The mothers and caretakers that do come for three follow-up visits are often characterized as those that are "eager to see their child gain weight", i.e., they recognize the importance of weight gain given the condition of the child (UNICEF personal communication).

This finding clearly shows that IPD and follow-up visits work as a program for treatment of SAM with complications. Our study emphasizes the importance of follow-up visits to further lower the WHZ and decrease the risk of relapse.

When looking at the progress and achievements in regard to IPD, treatment in hospitals has gone from a few days of hospitalization and low compliance regarding follow-up visits to an extended stay in hospital with a take-home ration of ready-to-use therapeutic foods (RUTFs) and improved compliance regarding follow-up visits. The increase found in the duration of stay in hospital might be explained by incentives provided to the caretakers, such as Health Equity Funds (HEF) [11]. The provision of HEF in regard to treatment of malnutrition was started in 2012 and are a pro-poor third party health financing mechanism providing health services for the identified poor (UNICEF personal communication). This means that the child is treated for free, including therapeutic foods, antibiotics, and any other drugs or supply needed. There is no charge for overnight stay, and they are provided with a food allowance per day. Additionally, the caretaker is reimbursed for transport fees, based on the distance and quality of the road. Transport support was also provided for follow-up visits; financial support and the introduction of a take-home ration are likely contributors to increased compliance [11].

In addition, the actual Cambodian guidelines and intervention emphasize the need to have a follow-up visit 5 weeks after discharge to avoid a relapse. In the context of Cambodia with a prevalence of wasting around $10 \%$, this final visit is essential to screen any tendency to prevent another episode of SAM (it seems not be the case for children having three follow-up visits). It is not yet well understood how much wasting contributes to conditions such as stunting. Evidence does suggest [12], however, that episodes of wasting negatively affect linear growth and, therefore, undermine child growth and development. It is, therefore, urgent to find a sustainable way to screen those children 5 weeks after; as of today only less than half of the children are coming back to these third visits.

\section{Limitations}

Nineteen hospitals over the 35 existing were assessed. The authors decided to assess the hospitals that have 
been implementing SAM for at least 3 years (since the national guideline was approved). The newly open hospitals have learned from those hospitals, and therefore, we used the hypothesis that they will perform better than the one trained previously. Severe acute malnutrition (SAM) is a nationwide issue with a national prevalence of $2.5 \%$ in 2010 [1]. The 13 provinces are homogeneous mixed of provinces with prevalence above and below the national prevalence for SAM.

The quality of the recording of patient files was poor in several hospitals. This might be due to a lack of training of staff or a lack of a proper system for recording. Therefore, we could not further analyze the use of the treatment (antibiotics, F75, F100, vitamin A, BP100) in the management of children with severe acute malnutrition in out-patient care. Even though there was limited recording of types of antibiotics received, one concern that has been aired is that antibiotics are given to all children, whether the child has SAM with complications or not thus increasing the risk of antibiotic resistance. There is a need to reeducate health care staff on proper use of antibiotics, and specify that if antibiotics are given it needs to be given consistently, the same type taken over a minimum of 10 days [13].

The collection of data on the treatment of SAM at the level of Hospitals did not appear to be standardized. Nutrition and treatment for SAM indicators have not yet been included in the Health Monitoring Information System. It is therefore urgent to develop a web-based monitoring system at hospital and health center level to ensure that the correct protocols are used and children are well-treated.

\section{Next steps}

Take-home rations are an important aspect of the treatment to ensure that the children will reach a WHZ $>-2$ $z$-scores. In order to be sustainable, the Government of Cambodia will have to include RUTF in their essential medical drug list to be able to purchase it and be less dependent on the funds of developing partners.

Capacities have been built in particular at the hospital, and attention has been paid to policy and guidelines, in particular the protocol for treatment of acute malnutrition. The limitation in terms of capacity development has been that much that the attention was focused at the individual level. Much less attention was paid to the institutional capacities, one of which concerns staffing structures, which leaves hospitals as well as health centers without staff with nutrition pre-service training. The establishment of an MSc course on Nutrition is an important step towards a more institutional approach to develop the capacities of health staffs and other individuals and can be expected to bear fruit only a few years from now.
With only 1600 cases annually referred to hospital and treated with F75, F100, and BP100 among an estimated 6000 cases of severe acute malnutrition with complications, mass screening is still a bottleneck as almost no routine screening is performed at the health center level or community level. In addition, unfortunately, the methodology used (mid-upper arm circumference (MUAC)) had a very small sensitivity and specificity to find the severely malnourished children [14]. It is essential to revise the guidelines for mass screening to ensure that children are well-screened.

\section{Conclusions}

Our findings suggest that the programs for treatment of malnutrition are working well, in regard to a higher proportion of children being cured than anticipated, despite the need for revision of the current guidelines to not use the gain weight as a discharge criteria. Those results should be used for advocacy purpose to ensure that the government invests more for the treatment of SAM as it seems to be well-implemented and getting positive results. In Cambodia, a short hospitalization with a prolonged follow-up may be a solution to improve nutritional status of severely malnourished children. That said, there is a need for an improved system; so by revising the current guidelines and providing refresher courses on the management including screening and treatment of SAM to the hospitals and health centers, further improvements might be seen on wasting prevalence and also on long term on stunting prevalence.

\section{Additional file}

Additional file 1: Figure S1. Provinces with hospitals interviewed (23 provinces with a total population of approximately 14 million individuals).

\section{Abbreviations}

SAM: severe acute malnutrition; UNICEF: United Nations children's fund; MDGF: millennium development goal funds; NNP: national nutrition program; WHZ: weight for height z-score; WAZ: weight for age z-score; ANOVA: one-way analysis of variance; IPD: in-patient treatment; SD: standard deviation; MUAC: mid-upper arm circumference; HEF: health equity funds; RUTF: ready to use therapeutic food.

\section{Competing interests}

The authors declare that they have no competing interests.

\section{Authors' contributions}

$\mathrm{SP}, \mathrm{MD}, \mathrm{SU}$, and $\mathrm{AL}$ conceived and designed the analysis. MD and SU collected the information. $A L$ and $M D$ analyzed the data. SP, MD, JC, AL. AL, JC, and SU wrote the paper and are or were UNICEF staff members. The opinions and statements in this article are those of the authors and may not reflect on the official UNICEF policies. All authors read and approved the final manuscript.

\section{Acknowledgements}

The authors acknowledge the National Nutrition Programme of Cambodia, cooperative health care staff at the 19 hospitals, and Sophal Chan for their on-going support during the assessment. We would also like to thank Frank Noij for his qualitative assessment on "the support to the Management of Acute Malnutrition at the level of the Hospital, the Health Center, and the 
Community in Cambodia" implemented in December 2013 for UNICEF and which has provided valuable information for the next steps. This research was conducted with the financial support of UNICEF National committees (Czech, Danish, Lithuanian, Norwegian, and Australian Committees).

\section{Author details}

${ }^{1}$ National Nutrition Program, Maternal and Child Health Center, No 31A, Rue de France (St. 47), 12202 Phnom Penh, Cambodia. ${ }^{2}$ UNICEF, Maternal,

Newborn and Child Health and Nutrition section, No. 11 street 75, 12202

Phnom Penh, Cambodia.

Received: 7 September 2014 Accepted: 27 May 2015

Published online: 22 June 2015

\section{References}

1. Harris S, Jack S. Home-based treatment of acute malnutrition in Cambodian urban poor communities. Food Nutr Bull. 2011;32(4):333-9.

2. United Nation. Joint Programme Children, Food Security, Nutrition. United Nation of Cambodia, 2013. [Online]. Available: http://www.mdgfund.org/ node/611. [Accessed 9 June 2015].

3. National Institute of Statistics. Cambodia Demographic and Health Survey 2010. Phnom Penh: National Institute of Statistics; 2011.

4. Bagriansky J, Champal N, Whitney S, Laillou A. The economic consequences of malnutrition in Cambodia, more than 400 million US dollar lost annually. Asian Pac J Clin Nutr. vol. in press, 2014.

5. National Nutrition Programme. National Interim Guidelines for the management of acute malnutrition. November 2011. [Online]. Available: http://camnut.weebly.com/policy-guidelines.html. [Accessed 15 July 2014].

6. Ballard M, Seng C, Conkle J. Monitoring malnutrition treatment compliance in Cambodia. Phnom Penh: UNICEF; 2013.

7. UNICEF. Early childhood nutrition fact sheet-Cambodia. Phnom Penh: UNICEF; 2013.

8. Bhutta Z, Das J, Rizvi A. The lancet nutrition interventions review group, and the maternal and child nutrition study group. Evidence-based interventions for improvement of maternal and child nutrition: what can be done and at what cost? Lancet. 2013;382(9890):396.

9. de Onis M, Onyango A, Borghi E, Garza C, Yang H. Comparison of the World Health Organization (WHO) child growth standards and the National Center for Health Statistics/WHO international growth reference: implications for child health programmes. Public Health Nutr. 2006;9:942-7.

10. WHO. Guideline: updates on the management of severe acute malnutrition in infants and children. Geneva: World Health Organization; 2013.

11. Ministry of Health. Standard benefit package and provider payment mechanism for health equity funds (Revised June 2012). Phnom Penh: Ministry of Health; 2012.

12. Khara T, Dolan C. The relationship between wasting and stunting, policy, programming and research implications. Washington D.C.: USAID Technical Brief Paper; 2014

13. Okeke IN, Aboderin OA, Byarugaba DK, Ojo KK, Opintan JA. Growing problem of multidrug-resistant enteric pathogens in Africa. Emerg Infect Dis. 2007;13(11):1640-6.

14. Laillou A, Prak S, de Groot R, Whitney S, Conkle J, et al. Optimal screening of children with acute malnutrition requires a change in current WHO guidelines as MUAC and WHZ identify different patient groups. PLoS One. 2014;9(7):e101159. doi:10.1371/journal.pone.0101159 Tables and Figures.

\section{Submit your next manuscript to BioMed Central and take full advantage of:}

- Convenient online submission

- Thorough peer review

- No space constraints or color figure charges

- Immediate publication on acceptance

- Inclusion in PubMed, CAS, Scopus and Google Scholar

- Research which is freely available for redistribution 